\title{
Naturalism and scientific creativity: new tools for analyzing science
}

\author{
Joke Meheus and Thomas Nickles (eds.): Models of discovery \\ and creativity. Dordrecht: Springer, 2009, x+249pp, €99, 95 HB
}

\section{Daniel Burnston}

Published online: 11 January 2011

(C) The Author(s) 2011. This article is published with open access at Springerlink.com

In the preface to Models of discovery and creativity, Thomas Nickles pitches the book as the continuation of a research program that began unofficially at a conference on scientific discovery in the late 1970's. The project is defined by its interdisciplinary nature, applying the combined resources of philosophy, social science, and cognitive science to the complex issues surrounding scientific concepts, practice, and methodology. Nickles offers the current volume as an update on the status of the program. All aspects of the project are present in the collected papers, which showcase an impressive array of analytical skills and historical expertise.

If read as a unified project, the aims of the contributors are extremely ambitious - the present volume suggests no less than an attempt to create a crossdisciplinary methodology for the analysis of scientific creativity and conceptual change. Analytical tools range from formal models of reasoning (Magnani, Sintonen), evolutionary computation (Nickles), and philosophical and cognitive theories of concepts (Brown, Nersessian); to detailed research into the daily experimental activities of scientists (Holmes), and analysis of personal cooperation and competition amongst researchers in contexts of discovery (Werner). The variety of scientific fields and episodes discussed borders on astounding, ranging from the discoveries of nuclear fission (Andersen) and messenger RNA (Darden), to the construction of concepts and proofs in geometry and algebra (Glas).

At first glance, it would seem impossible that experts in such a variety of fields should be employing anything like a unified methodology, or approaching a unified goal. It might be suspected that the volume is simply a grouping of a variety of really independent projects analyzing science from the distinct fields of philosophy of science, cognitive science, and science studies. However, there is a shared

\footnotetext{
D. Burnston $(\bowtie)$

Department of Philosophy, Interdisciplinary Cognitive Science Program, University of California, San Diego, 9500 Gilman Drive \# 0119, La Jolla, CA 92093-0119, USA e-mail: dburnsto@ucsd.edu
} 
perspective to be gleaned from the volume, which involves a shared commitment to assessing science from an empirical viewpoint. Let us call this the 'naturalism' perspective. The goal of the naturalism perspective is to give detailed accounts of science that reflect the on-the-ground practices of scientists. This entails replacing a number of cherished philosophical habits with the analytical tools mentioned above. One such abandoned habit is that of construing science entirely in terms of abstract theories individuated by their logical structure. ${ }^{1}$ Several authors (Darden, Glas) also repudiate the distinction between discovery and justification (Nickles, p. 190, is a possible exception), a standard viewpoint that has often allowed philosophers to ignore the practical elements of scientific progress in favor of a logical analysis of the justification for holding a particular theory. One of the recurring themes of the book is that, under the naturalism perspective, there are no such clear distinctions to be made in analyzing actual scientific practice-any phenomenon of scientific reasoning is going to be complex and multi-faceted, and real understanding how science works depends on an understanding of actual scientists in actual cognitive and social contexts. The commitment to cross-disciplinary projects and historical analysis stems from this position.

Laudably, this shared commitment supplies us with several well-thought-out frameworks for analyzing both (i) the conceptual structures involved in scientific thought; and (ii) the practices of constructing explanations and discovering anomalies. The most well-developed proposal for (i) is provided by Nersessian, who for several years has been promoting a view of scientific concepts inspired by Johnson-Laird's (1983, 2004) account of 'model-based reasoning' (MBR). As applied to science by Nersessian, MBR claims that scientific concepts are iconic representations of a target domain that embody structural constraints isomorphic (or homomorphic) to those present in that domain. Reasoning with models consists of running representational 'simulations' (p. 141) of the behavior of the target domain. Creativity and concept change are processes of modifying the constraints present in the representational model so as to produce different behavior in simulation.

Brown provides an alternate view based on Sellars' theory of empirical concepts. On the Sellarsian view, concepts are characterized by 'entry transitions' and 'local holisms'. An entry transition is the cognitive process of transforming an ability to recognize an item or class into the ability to make inferences about the item or class. Local holisms are the particular set of inferences that are drawn in relation to the concept. On this scheme, concept change consists in modifying the set of inferences that one is disposed to make with the concept. Finally, Andersen reprises Kuhn's theory of categorization by similarity class, on which concepts are feature lists that are used to group similar objects together. Change consists in modifying the feature lists to produce new taxonomies. While these theories differ both in focus and in implications, it is clear that they share a commitment to explaining scientific concepts in a naturalistic way.

\footnotetext{
${ }^{1}$ Even the broadly model-theoretic perspective offered by Sintonen emphasizes the hierarchical and flexible nature of theories, and recognizes (although not unreservedly) the relevance of cognitive science for understanding creativity and conceptual change (see p. 213).
} 
Element (ii) of the naturalism perspective deals with hypothesis generation, theory construction, anomaly resolution, and discovery. ${ }^{2}$ Once again, a number of useful frameworks are to be found. Darden argues that giving explanations in biology consists of constructing 'mechanism sketches' - abstract representations that specify the functional or causal roles in a mechanistic system-and then 'filling in' the sketch with entities and sub-processes to produce a completed 'mechanism schema'. Anomalies are unfulfilled or unclear roles in a mechanism schema, and discovery occurs when an entity is specified that solves the anomaly. Quite strongly, Darden argues that mechanism schemas simply are the theories that biologists construct.

Abductive reasoning is a focus of several of the essays. One of the persistent criticisms of positivistic and deductive views of science has been that purely deductive systems leave the generation of new content a complete mystery. Both Kleiner and Magnani attempt to fill this theoretical gap via abduction, which is an ampliative type of inference, in that it generates novel content out of possessed content. On Kleiner's view, abduction is a constraint on hypothesis generation, in which one's previously possessed concepts and explanations both provide an analogical basis for new hypotheses and limit the range of plausible hypotheses by generating abstract principles (e.g., about types of causes and the properties of natural kinds) which can be applied to new domains. Novelty then emerges from the new applications. Magnani's focus is on incorporating abduction within a broader view of scientific epistemology. He argues that, in addition to generating new hypotheses, abduction also forms the basis for inference to the best explanation. Both accounts point out that while there may be no 'logic' to discovery, this does not make it intractable, as abduction is a reasoned process that can be reconstructed with careful analysis.

Nickles gives a more deflationary account of theory change, relying both on Popper's evolutionary epistemology and on results from research on evolutionary computation. Evolutionary epistemology argues that the generation of new hypotheses parallels the generation of new phenotypes, in that it consists of random generation of new material that is then selectively retained due to producing some degree of fitness (in this case, explanatory value). Evolutionary computation has shown that successive random re-combinations of computer code, when combined with a parameter for fitness and a mechanism of selective retention, can eventually produce programs capable of solving even sophisticated engineering problems. Nickles argues that scientific progress occurs in largely the same way. While once again there are differences between these proposals, they clearly share the naturalism perspective.

Where does the project stand, then, as a unified approach to understanding science? A nebulous shared perspective, interesting though it may be, does not a methodology make, and a number of obstacles stand between the current project and a unified viewpoint. The first, of course, is the very breadth of tools and phenomena under discussion. It is often unclear how the accounts offered here fit together, even in cases where it seems intuitive that they would do so. For instance, is Darden's process of mechanistic explanation realized cognitively by something like

\footnotetext{
2 Unsurprisingly, this element is closely related to (i). For instance, Andersen attempts to account for the generation of anomalies and the difference between expected and unexpected discoveries via the amount and type of revision that they demand of an existing taxonomic framework. However, I separate these aspects from element (i) because their focus is more on practice than on conceptual structure or content.
} 
Nersessian's MBR view? This would seem to be a reasonable position, but the question is not addressed, and it is unclear that the model-based view (as compared to, say, an inferentialist view such as Brown's) is the only or best cognitive account to conjoin with Darden's theory of explanatory practice.

A similar concern arises in the papers that discuss specific scientific episodes. The structure of several of these is to offer and explain a framework, F, for understanding some aspect of science, followed by an illustration of some episode, E, from the history of science, which it is supposed to elucidate or explain. But the fact that one can justifiably apply $\mathrm{F}$ to $\mathrm{E}$ at best shows that $\mathrm{F}$ is a possible way of understanding $\mathrm{E}$, not that it is the only or best way. Alternate models are rarely discussed in the individual papers. This is to say nothing of cases in which the accounts straightforwardly disagree. As already mentioned, Nickles' evolutionary framework disagrees with the abductive views of Kleiner and Magnani on the level of randomness involved in theory change. To take another example: Nersessian endorses a 'continuum hypothesis' (p. 128), on which scientific concepts and reasoning are on a continuum with their everyday counterparts, while Brown argues that we have distinct conceptual systems for folk and theoretical modes of reasoning. Examples could be added, but these suffice to show that there are deep differences between the accounts on offer here.

What these considerations suggest is that the research program is still in its formative stages, despite Nickles' announcement of its 30th anniversary. If the current volume is any indication, the attention is still on constructing the tools necessary for this type of analysis-tools that take the naturalism perspective seriously and attempt to combine the resources of multiple disciplines in its pursuit. Given the thoughtfulness and depth of the models on offer in the book, it seems as though this stage is nearing fruition. What remains, then, is to compare the frameworks presented, arguing out both their internal differences and their possible applications. Of course, once this work is embarked on, there may turn out to be competing methodologies within the naturalism perspective, perhaps emerging from different ways of combining and prioritizing the current models. This would be an undeniably good thing, as it would foster specific, issue-focused dialectic that would further the understanding of the entire perspective.

Far from being a downfall of the book, these issues show that it succeeds as an excellent window into the state of a broad theoretical endeavor-the understanding of science from the naturalism perspective. The book contains a wealth of interesting models and questions for further consideration. If the field is not yet mature, it has at least reached the point where we can be excited about the potential it holds.

Open Access This article is distributed under the terms of the Creative Commons Attribution Noncommercial License which permits any noncommercial use, distribution, and reproduction in any medium, provided the original author(s) and source are credited.

\section{References}

Johnson-Laird, P. 2004. The history of mental models. In Psychology of reasoning: Theoretical and historical perspectives, eds. K. Manktelow, and M. Cheung Chung, 179-212. New York: Psychology Press.

Johnson-Laird, P. 1983. Mental models. Cambridge, MA: Harvard University Press. 